\title{
The Association between Factor XI Deficiency and the Risk of Bleeding, Cardiovascular, and Venous Thromboembolic Events
}

\author{
Sarah Sharman Moser ${ }^{1}$ Gabriel Chodick ${ }^{1,2}$ Yan G. $\mathrm{Ni}^{3}$ Dan Chalothorn ${ }^{3}$ Ming-Dauh Wang ${ }^{3}$ \\ Alan R. Shuldiner ${ }^{3}$ Lori Morton ${ }^{3}$ Ophira Salomon ${ }^{2,4}$ Jessica J. Jalbert ${ }^{3}$
}

\footnotetext{
${ }^{1}$ Maccabitech, Maccabi Institute for Research and Innovation, Maccabi Healthcare Services, Tel Aviv, Israel

2 Sackler Faculty of Medicine, Tel Aviv University, Tel Aviv, Israel

3 Regeneron Pharmaceuticals, Inc., New York, United States

${ }^{4}$ Institute of Thrombosis and Hemostasis, Sheba Medical Center, Tel

Hashomer, Ramat Gan, Israel
} Address for correspondence Jessica J. Jalbert, PhD, 777 Old Sawmill
River Road, Tarrytown, NY 10591, United States
(e-mail: Jessica.Jalbert@Regeneron.com).

Thromb Haemost 2022;122:808-817.

\begin{abstract}
Keywords

- factor XI

- bleeding risk

- cardiovascular events

The objective of this study was to assess the relationship between factor XI (FXI) deficiency and the risks of bleeding and cardiovascular (CV) events. We conducted a retrospective cohort study using data from Maccabi Healthcare Services (MHS). We identified adults with FXI deficiency (severe: $<15 \%$, partial: 15 to $<50 \%$, any deficiency: $<50 \%$ ) that had been tested for FXI between 2007 and 2018 and matched to patients from the general MHS population. We estimated 10-year risks of outcomes using the Kaplan-Meier approach. Using Cox proportional hazards regression, we compared outcomes among patients with versus without FXI deficiency. Less than $10 \%$ of patients tested for $\mathrm{FXI}$ activity had activity levels $<50 \%$ (mean age: 39 years; $72.2 \%$ females). Compared with the general population, patients with any FXI deficiency were at higher risk of severe bleeding (adjusted hazard ratio [aHR]: 2.56, 95\% confidence interval [CI]: $1.13-5.81$; 10 -year risk: $1.90 \%, 95 \% \mathrm{Cl}: 0.50-3.20 \%$ vs. $0.90 \%, 95 \% \mathrm{Cl}: 0.50-1.30 \%$ ) and clinically relevant nonsevere bleeding (CRNSB) (aHR: 1.45, 95\% Cl: 1.08-1.97; 10-year risk: $11.60 \%, 95 \% \mathrm{Cl}: 8.30-14.80 \%$ vs. $9.20 \%$, 95\% Cl: $8.00-10.40 \%)$. Severe FXI deficiency was associated with a greater risk of CRNSB. While few CV events $(N=2)$ and venous thromboembolisms (VTE) $(N=1)$ were observed in the FXI overall deficient group, there was a nonsignificant negative association between any FXI deficiency and CV events (aHR: 0.55; 95\% Cl: 0.13-2.36) and VTEs (aHR: 0.45; 95\% Cl: 0.06-3.47). Overall FXI deficiency was associated with an increased risk of severe bleeding and CRNSB. Further research is warranted to explore the lower risk of CV and VTE among patients with FXI deficiency compared with the general population.
\end{abstract}

Factor XI (FXI) deficiency is a mild bleeding disorder first described in the early 1950s, which affects both males and females equally. ${ }^{1,2}$ It was identified as an autosomal recessive disorder with a prevalence of one per million worldwide, and a much higher prevalence among Ashkenazi Jews (as high as 1 per 450) due to homozygosity or compound heterozygosity for two founder mutations in F11. ${ }^{3-7}$ received

May 30, 2021

accepted after revision

July 21, 2021

published online

September 23, 2021
DOI https://doi.org/

$10.1055 / \mathrm{s}-0041-1735971$. ISSN 0340-6245.

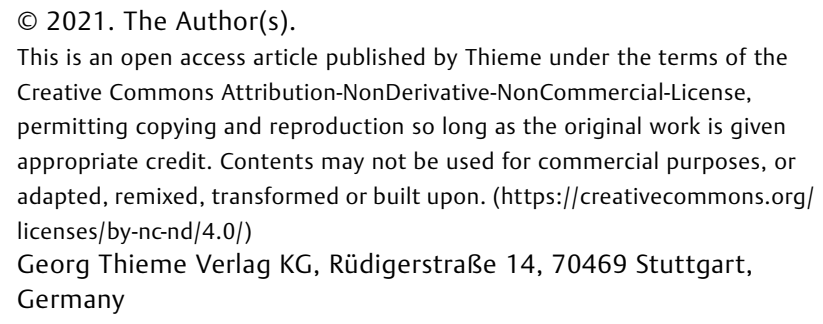


FXI deficiency is characterized by prolonged bleeding after trauma or surgery and patients rarely exhibit spontaneous bleeding typical of other hemophilia diseases. ${ }^{2,8,9}$ The correlation between FXI activity levels and bleeding, however, has not been well established ${ }^{10,11}$; some studies have reported that low levels of FXI do not predict clinical bleeding severity ${ }^{12,13}$ while other studies have found that patients with severe FXI deficiency had increased bleeding in tissues exhibiting higher fibrinolytic activity such as the oral cavity, tonsils, nose, and urinary tract. ${ }^{89}$ There is also some evidence that FXI deficiency may be protective against some thromboembolic events. Studies have shown that patients with severe FXI deficiency $(<15 \%)$ may have a reduced risk of ischemic stroke (cerebrovascular accident [CVA] $)^{14}$ and venous thromboembolism (VTE), ${ }^{15-17}$ while patients with an increase in FXI levels have increased risk of ischemic stroke ${ }^{18}$ and deep vein thrombosis. ${ }^{19} \mathrm{~A}$ recent study also found dramatic reductions in the risk of cardiovascular (CV) events (defined as stroke, transient ischemic attack [TIA], and myocardial infarction [MI]) and VTE among patients with FXI deficiency (i.e., FXI activity $\leq 50 \%$ ) compared with patients with normal FXI levels (i.e., $>50 \%$ ). ${ }^{17}$

Questions remain, however, regarding the relationship between bleeding outcomes among patients with partial deficiency $(15$ to $<50 \%)$, and whether inducing partial deficiency using therapeutic agents could protect from CV events without increasing risk of bleeding. The objective of this study was to assess the relationship between FXI deficiency and the risks of bleeding (severe bleeding and clinically relevant nonsevere bleeding [CRNSB]) and CV events in a large health care insurance provider with one of the largest populations of FXI-deficient individuals in the world.

\section{Methods}

\section{Data Source}

This matched retrospective cohort study was conducted using the computerized databases of Maccabi Healthcare Services (MHS), a nationwide health care insurer-provider representing a quarter of the population in Israel, with data on 2.4 million members currently. The MHS database was established in 1993 and contains longitudinal data on a stable population (with less than $1 \%$ yearly attrition). Data are automatically collected and include complete capture of inpatient and outpatient care, comprehensive laboratory data from a single central laboratory, full outpatient pharmacy prescription and dispensation data, and extensive demographic data on each patient. MHS couples the International Classification of Diseases, Ninth Revision, Clinical Modification (ICD-9-CM) coding system with internal coding systems to provide more granular diagnostic information beyond the ICD codes. Medications are coded according to the Israeli coding system with mapping to Anatomical Therapeutic Chemical classification system whenever available. Procedures are coded using Current Procedural Terminology codes. MHS has developed several registries of major chronic diseases, such as CV disease, oncologic diseases, diabetes mellitus, kidney disease, and osteoporosis, to improve the quality of chronic care delivery to its members. ${ }^{20-24}$ The registries are continuously updated, and they identify patients via automatic search algorithms, as opposed to being dependent upon active reporting by physicians.

\section{Study Population and Design}

We identified patients' first FXI blood test conducted in the outpatient setting between January 1, 2007 and December 31,2018 . While earlier data were available, we included patients with laboratory testing on or after 2007 because testing was centralized at MHS as of 2007. The date of the FXI test was defined as the index date. For each patient with a FXI test, we matched seven patients who were insured by MHS but not tested for FXI (i.e., general population reference group [GPRG]) who had an outpatient visit or a drug dispensation within 3 months of the FXI test date (to ensure that the patient was an active MHS patient), on birth year, sex, and health clinic. GPRG matches were assigned the same index date as their FXI patient.

To be eligible for the study, patients were required to be aged 18 years or above on the index date, enrolled at MHS for at least 1 year, and not have a history of cancer (except nonmelanoma skin cancers). In addition, for all outcomes except CRNSB, patients who had experienced the event prior to the index date were excluded. We therefore had four separate study cohorts to analyze different outcomes: (1) full study cohort including all patients with a FXI test and their GPRG matches, regardless of whether they had previously experienced outcomes (cohort used to describe patients at baseline), (2) patients without a prior severe bleed, (3) patients without a prior CV event, and (4) patients without a prior VTE event.

\section{FXI Activity Levels}

FXI levels were categorized as severe ( $<15 \%)$, partial (15 to $<50 \%$ ), overall deficient $(<50 \%$, including both severe and partial deficiency), or normal FXI activity ( $\geq 50 \%$ ). The categorization of FXI activity was based on research demonstrating that patients who are homozygous or compound heterozygous for the FXI mutations have FXI activity levels less than $15 \%$, patients with FXI activity between $15 \%$ but less than $50 \%$ are considered to have partial deficiency, and patients with FXI activity $\geq 50 \%$ are considered to have normal FXI activity levels. ${ }^{25}$

\section{Outcomes and Follow-Up}

We identified severe bleeding using validated ICD-9-CM code lists ${ }^{26-28}$; code position could not be applied as discharge diagnoses are not ordered in Israel. Severe bleeding was defined as a hospitalization with a diagnosis of any of the following: hemorrhagic stroke (positive predictive value $[\mathrm{PPV}]>85 \%$ ), severe (upper, lower, or unspecified) gastrointestinal bleed (PPV: 85.8-87.8\%), severe urogenital bleed (PPV: 100\%), or other bleed (PPV: 95.5\%), which included hemopericardium, unspecified hemorrhage, nontraumatic hemoperitoneum, hemarthrosis, epistaxis, hemorrhage from the throat, and hemoptysis. We identified CRNSBs using the same codes as severe bleeding; however, for the 
event to be considered CRNSB, we required at least two outpatient codes (for validation purposes) on different days for the same type of bleed without hospitalization; or a bleeding diagnosis within 7 days of a transfusion and no hospitalization 30 days prior to the diagnoses or transfusion. Hemorrhagic stroke could not be considered a CRNSB.

We identified MI, CVA, or TIA events using validated MHS registries, which identify patient's first lifetime event according to an established algorithm. ${ }^{20}$ Since there is no MHS registry for VTE, we used an ICD-9-CM algorithm with a sensitivity of $72 \%$, specificity of $99 \%$, PPV of $91 \%$, and negative predictive value of $95 \%$ to identify VTEs. ${ }^{29}$ The algorithm included requiring a diagnostic code for VTE in the inpatient or outpatient setting with at least one of the following within 30 days of diagnosis: a pharmacy prescription for anticoagulation, placement of an inferior vena cava filter, or death.

Individuals were followed from the index date until the occurrence of the outcomes of interest, death, loss to followup, incident nonmelanoma cancer diagnosis, or end-of-study period (December 31, 2019), whichever occurred first.

\section{Study Variables}

Socio-demographic data were categorized according to their value at the index date: age (continuous and $>65$ years), sex (male/female), Israeli district (Center, South, North), and socioeconomic status (SES: low, medium, high). ${ }^{30}$ Smoking status was classified as current/past or never. To estimate body mass index (BMI), we selected the most recent BMI value recorded in a patient's record within 5 years prior to index date.

To identify patients with diabetes mellitus, hypertension, ischemic heart disease, atrial fibrillation, MI, cerebrovascular disease, and chronic kidney disease, we used disease-specific MHS registries. ${ }^{20,22,23}$ For hyperlipidemia and chronic obstructive pulmonary disease, patients with two or more ICD9-CM codes before index date were categorized as having the disease. Cancer history was obtained from the MHS cancer registry which uses diagnoses linked to cancer medication approvals and pathology reports. ${ }^{24}$ The Deyo-Charlson Comorbidity Index, using ICD-9-CM codes and MHS registries to determine presence/absence of disease was calculated. ${ }^{31}$

We identified exposures to medications that could affect risk of bleeding and/or CV events, including: antiplatelets, anticoagulants (i.e., vitamin $\mathrm{K}$ antagonists [VKAs], heparin, low-molecular-weight heparins [LMWHs], direct-oral anticoagulants), nonsteroidal anti-inflammatory drugs, and antipsychotic drugs. Patients were considered to have received therapy if there were at least two dispensations 6 months prior to the index date.

To estimate bleeding risk, we estimated the HAS-BLED risk score $^{32}$ (although the international normalized ratio would not have been assessed for most patients) and for atrial fibrillation stroke risk, we estimated the $\mathrm{CHA}_{2} \mathrm{DS}_{2}$-VASc score. ${ }^{33}$

\section{Statistical Analysis}

We report socio-demographic characteristics, baseline comorbidity burden, laboratory test results, and prescription medication among patients with severe ( $<15 \%)$, partial (15 to $<50 \%$, and any FXI deficiency $(<50 \%)$ and their matched GPRG. We report numbers and proportions for categorical variables and, for continuous variables, the mean and standard deviation.

The Kaplan-Meier approach was used to estimate the 10year cumulative incidence and 95\% confidence intervals (CIs) of the first severe bleeding, CRNSB, CV, and VTE events over follow-up among patients with severe, partial FXI deficiency, and any FXI deficiency and their GPRG matches.

Cox proportional hazards regression models were used to derive unadjusted and adjusted hazards comparing the risk of outcomes among patients with $<15 \%, 15$ to $<50 \%$, and $<50 \%$ FXI activity to their GPRG. Propensity scores were used to adjust for confounding by including the propensity score as a continuous variable in the Cox model. A separate propensity score was derived for each comparison (e.g., $<15 \%$ FXI vs. matched GPRG) and included the following variables: age (continuous), sex, district, SES, smoking status, BMI (continuous), Deyo-Charlson Comorbidity Index (continuous), comorbidities, drug exposures in the past 6 months, the HAS-BLED (for bleeding), and the $\mathrm{CHA}_{2} \mathrm{DS}_{2}$-VASc score (for CV outcomes).

\section{Sensitivity Analyses}

We conducted several sensitivity analyses to assess the robustness of the results. To replicate the Preis et al's study, ${ }^{17}$ we compared the risk of severe bleeding, CV, and VTE events between patients with FXI deficiency ( $<15 \%, 15$ to $<50 \%$, and $<50 \%$ ) and those with normal FXI activity (i.e., patients with a FXI activity test results of $\geq 50 \%$ ). We also assessed the association between FXI activity and prevalent (vs. incident) severe bleeding, CV, and VTE events by not restricting the study population to patients without a prior event and identifying the first event after index date, using validated published algorithms. ${ }^{34,35}$ In addition, we used the cut point of $<20 \%$ to define severe FXI deficiency because of the difference in how some researchers define severe deficiency. ${ }^{2,13,36}$ Lastly, we explored the impact of left truncation bias. Since FXI deficiency is present from birth and the index date is the date of FXI test, a random point in time, potential biases may stem from missing information for the time period prior to the index date. Left truncation bias could arise if patients have a fatal CV event prior to having a FXI test because they would not be included in the FXI group. To assess whether truncation time varies differentially between groups due to a difference in mortality rate, we calculated the number of deaths 3 months after a CV event for FXI patients and their matched GPRG.

The study was approved by the local ethics review board of MHS in Israel. All analyses were conducted using IBM SPSS Statistics for Windows, Version 22.0. Armonk, New York: IBM Corp.

\section{Results}

We identified 7,578 patients with a FXI test and 7,403 patients were successfully matched to seven GPRG patients on birth year, sex, and health clinic (-Supplementary Table S1, available in the online version). Over $90 \%$ of patients ( $n=6,817)$ 
with a FXI test had normal activity levels, 5.0\% $(n=373)$ had partial deficiency, $2.9 \%(n=213)$ had a severe deficiency, and $7.9 \%(n=586)$ had any deficiency in FXI activity (-Supplementary Fig. S1 [available in the online version] and - Table 1). Distribution of patients was similar across cohorts without prior severe bleeding, CV, or VTE events (-Supplementary Table S2, available in the online version).

Mean age at index date for patients with FXI deficiency was 39 years, $7 \%$ were over the age of 65 years, and almost 75\% were females (-Table 1). Patients with severe FXI deficiency were on average approximately 10 years older, were less likely to be female, had a higher comorbidity burden, and had a higher bleeding score than patients with partial FXI deficiency.

Overall, patients with FXI deficiency and GPRG were well matched on socio-demographic variables (-Table 1). Comorbidity burden, medication use, and risk scores were also generally well balanced among patients with FXI deficiency and the GPRG. Fewer patients with FXI deficiency were current or past smokers compared with their GPRG matches.

\section{Bleeding Events}

Over a median follow-up of 8.00 years (Q1-Q3: 4.12-9.98) among FXI-deficient patients and 7.98 years (Q1-Q3: 4.129.95) among the GPRG, there were eight events of severe bleeding observed in the FXI-deficiency group compared with 22 in the GPRG (- Table 2 and - Fig. 1). Severe bleeding in the FXI-deficiency group included two patients with bleeding after rhinoplasty, irregular menstrual bleeding with hospitalization, posthemorrhagic anemia after liver transplant, rectal bleeding after colonoscopy and polypectomy, lower gastrointestinal bleed after colonoscopy (also receiving LMWH), gastrointestinal bleed (also receiving VKA), and subarachnoid hemorrhage (also receiving antiplatelet therapy).

The 10 -year cumulative incidence $(95 \% \mathrm{CI})$ of severe bleeding among patients with FXI deficiency (1.9\%; 95\% CI: $0.5-$ $3.2 \%$ ) was more than twice that of patients in the GPRG $(0.9 \%$; 95\% CI: 0.5-1.3\%), even when matched on birth year, sex, and health clinic. The risk of severe bleeding among patients with FXI deficiency remained elevated compared with GPRG after adjusting for confounders (adjusted hazard ratio $[\mathrm{aHR}]=2.56$; 95\% CI: 1.13-5.81). When stratified by severity of FXI deficiency, compared with patients in the GPRG, partial FXI deficiency was strongly associated with an increase in severe bleeding, $(\mathrm{aHR}=5.27 ; 95 \% \mathrm{CI}: 1.91-14.52)$ but severe FXI deficiency was not $(\mathrm{aHR}=0.49 ; 95 \% \mathrm{CI}: 0.06-3.95)$.

For CRNSB, the median follow-up time was 7.46 years (Q1-Q3: 3.51-9.80) and 7.57 years (Q1-Q3: 3.67-9.80) among the FXI-deficient patients and the GPRG, respectively. The 10-year cumulative incidence of CRNSB was also higher among patients with FXI deficiency than among the matched GPRG (11.6\%; 95\% CI: 8.6-14.8\% vs. 9.2\%; 95\% CI: $8.0-10.4 \%$ ) (-Table 2 and -Fig. 1). After confounder adjustment, patients with FXI deficiency remained at higher risk than their matched GPRG counterparts (aHR: 1.45 ; $95 \%$ CI: $1.08-$ 1.97). The 10-year cumulative incidence was higher among patients with severe FXI deficiency (13.8\%; 95\% CI: 7.7$19.6 \%$ ) than among patients with partial deficiency (10.5\%;
95\% CI: 6.6-14.3\%). The aHR for FXI deficiency compared with GPRG was 1.53 (95\% CI: 0.94-2.47) for severe deficiency and 1.41 (95\% CI: 0.95-2.08) for partial deficiency.

\section{CV/VTE Events}

We observed few CV events over the study period, particularly in the FXI-deficiency group (-Table 3 and -Fig. 2). There were two CV events in the FXI-deficiency group and 23 events in the GPRG over a median follow-up time of 7.93 years (Q1-Q3:4.01-9.94) and 7.95 years (Q1-Q3:4.079.94), respectively. The 10 -year cumulative incidence was lower among patients who had FXI deficiency than among the GPRG: $0.4 \%$ (95\% CI: $0.0-0.9 \%$ ) versus $0.9 \%$ (0.5-1.2\%), respectively. While not significant, the point estimate suggests that FXI deficiency may have a negative association with CV events compared with GPRG $(\mathrm{aHR}=0.55 ; 95 \% \mathrm{CI}$ : $0.13-2.36$ ). We did not observe a correlation between severity of FXI deficiency and a reduction in CV events.

We also observed few VTEs over follow-up (median follow-up time in years was 7.90 years [Q1-Q3: 3.96-9.93] for patients with overall FXI deficiency vs. 7.93 years [Q1Q3:4.03-9.93] among the GPRG); there was one event in the FXI-deficient cohort and 13 in the GPRG (- Table 3 and -Fig. 2). Ten-year cumulative incidence of VTEs was lower among patients with FXI deficiency $(0.2 \%$; $95 \% \mathrm{CI}$ : $0-.60 \%)$ than among the GPRG $(0.5 \%$; $95 \% \mathrm{CI}: 0.2-0.7 \%$, $p=0.54)$. While not significant, we found a negative association when we compared risk of VTEs among patients with FXI deficiency to GPRG (aHR $=0.45 ; 95 \% \mathrm{CI}: 0.06-3.47)$. The only VTE observed among patients with FXI deficiency occurred among those with partial FXI deficiency; no VTEs were observed among patients with severe FXI deficiency.

\section{Sensitivity Analyses}

In one of the sensitivity analyses, we compared CV outcomes among patients with FXI deficiency to patients with normal FXI activity levels ( $\geq 50 \%$ ). We first explored the relationship between FXI deficiency testing and outcomes and found an increased risk of each outcome among patients who had normal FXI activity levels compared with their GPRG, even after confounder adjustment (-Supplementary Fig. $\mathbf{S 2}$ and -Supplementary Table S3, available in the online version). Next we compared outcomes between patients with FXI deficiency (i.e., $<50 \%$ ) to patients with normal FXI activity levels (i.e., $\geq 50 \%$ ). While results were generally directionally consistent, i.e., FXI deficiency was associated with increased risk of bleeding (except for CRNSB where there was no association) and a decreased risk of CV and VTEs, the choice of control group impacted results (-Supplementary Fig. \$3 and -Supplementary Table S4, available in the online version). Notably, when patients with a normal FXI test result were used as the reference group, the association between FXI activity and bleeding was attenuated and the protective association between FXI and CV and VTEs was increased.

The results of sensitivity analyses estimating the association between FXI activity levels and prevalent severe bleeding and CV events were consistent (-Supplementary Tables $\mathbf{S 5}$ and S6, available in the online version), except that, either 


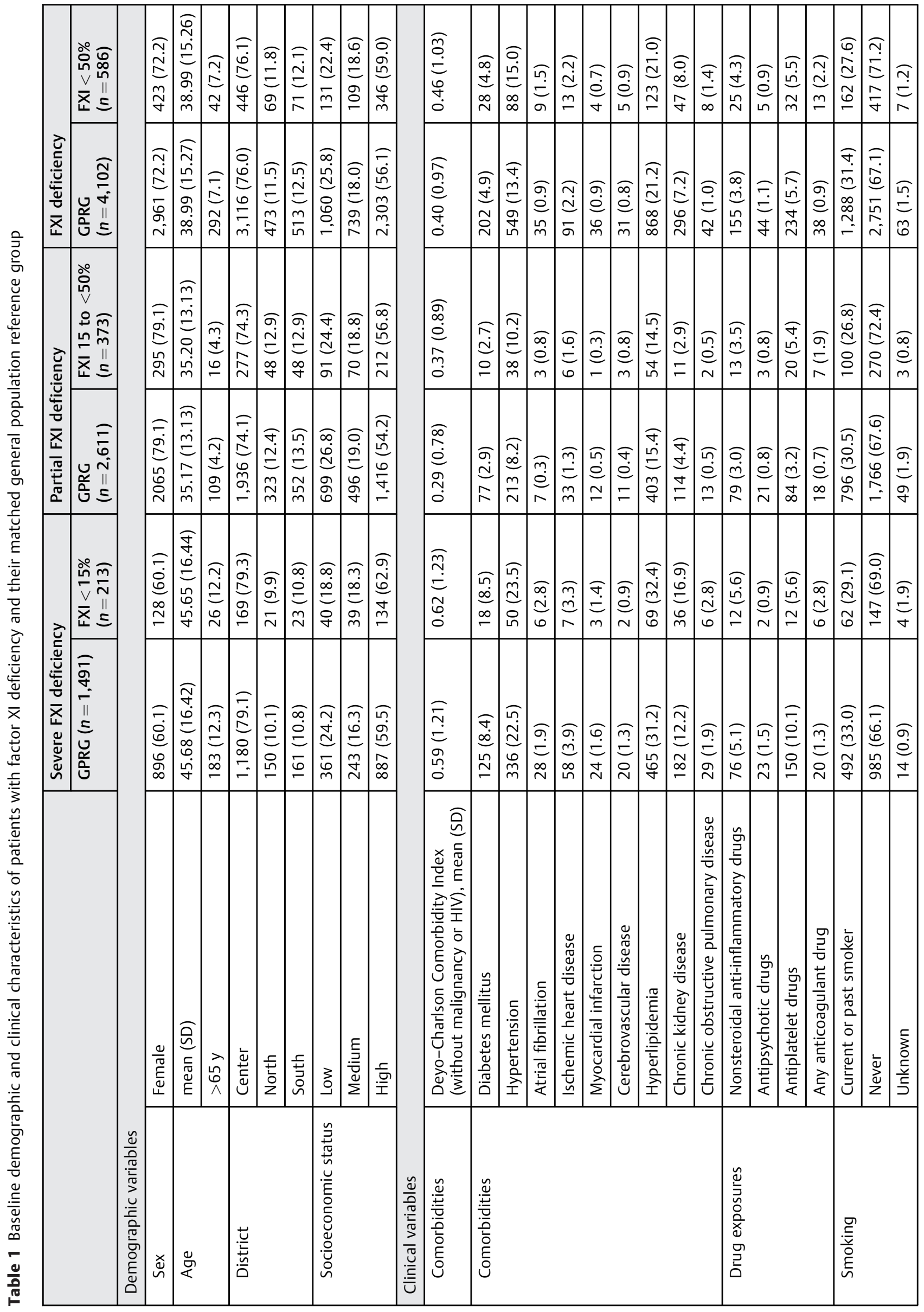




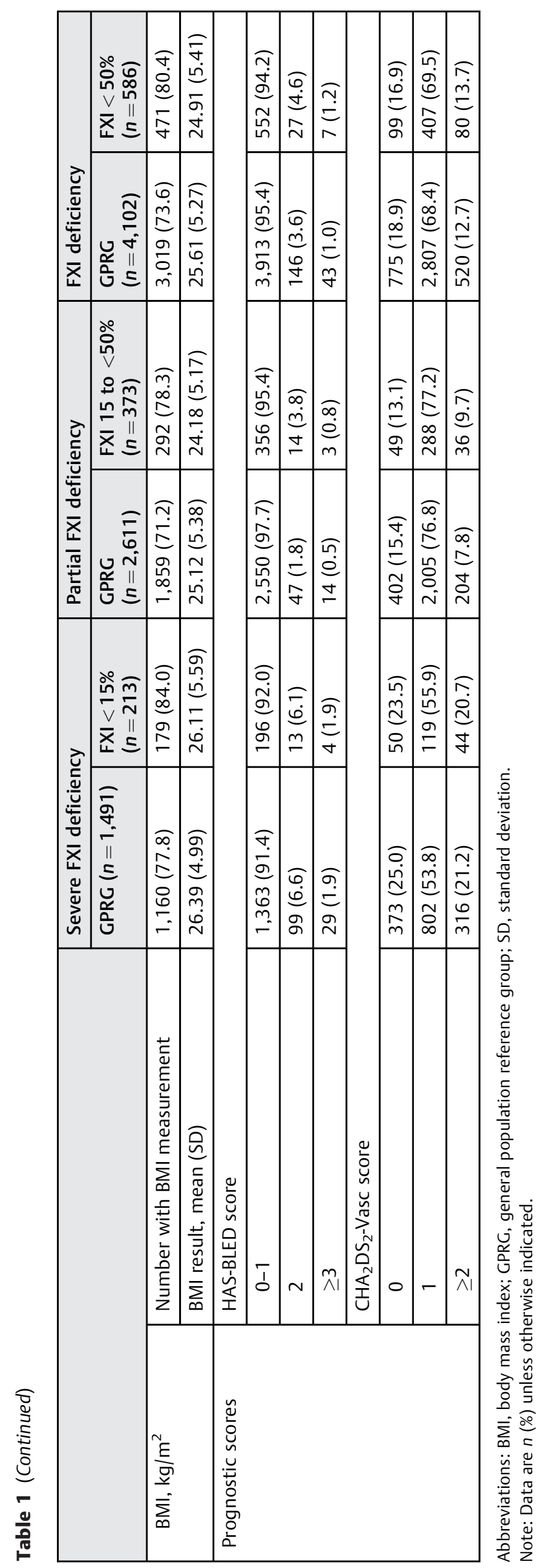

due to chance or small numbers, patients with FXI deficiency had a higher 10-year risk of prevalent VTE than the GPRG (aHR: 1.13; 95\% CI: 0.32-3.96) (-Supplementary Table 57, available in the online version). Results were robust when severe FXI activity was defined as $<20 \%$ and partial deficiency was defined as being between 20 and $<50 \%$. Results exploring the potential effect of left truncation bias showed no differences in case fatality among patients with FXI deficiency or GPRG, suggesting nondifferential truncation time between the groups.

\section{Discussion}

In this study we found that less than $10 \%$ of tested patients were FXI-deficient. We also found that FXI deficiency was associated with an increased risk of both severe bleeding and CRNSB. Patients with severe FXI deficiency were at greater risk of CRNSB than patients with partial FXI deficiency. While few CV and VTE events were observed among patients with FXI deficiency and, as a result, Cls were wide, our findings also suggest a negative association between FXI deficiency and CV and VTE events.

In this study we found an increased bleeding risk among patients with FXI deficiency for both severe bleeding and CRNSB similar to previous studies. ${ }^{9,37}$ We found that most of the cases of severe bleeding in patients with partial deficiency were postprocedural, thereby confirming that patients with FXI deficiency are unlikely to bleed spontaneously. ${ }^{2}$ Unlike other hemophilia diseases, an increased risk of bleeding in patients with partial FXI deficiency has not been well established, ${ }^{10}$ nor a dose-response relationship for risk of bleeding by FXI level. ${ }^{2,12}$ Patients with FXI deficiency may be more likely to be sent for FXI testing due to a prolonged partial thromboplastin time result routinely checked prior to surgery. The lack of a dose-response between FXI activity levels and bleeding risk may be explained in part by increased preoperative screening and prophylactic treatment among FXI-deficient patients undergoing surgery and avoidance of platelet inhibitors and nonsteroidal anti-inflammatory agents. ${ }^{38}$

While few CV events and VTEs were observed in the FXIdeficient group, the point estimates suggest that FXI deficiency may have a negative association with CV and VTE events. This is consistent with several studies which found that patients with severe FXI deficiency have a reduced risk of $\mathrm{CV}^{14}$ and $\mathrm{VTE}^{15-17}$ events. Moreover, the point estimates from our study were very similar to those of a recent study ${ }^{17}$ in a similar population. The aHR for CV events in the previous study was 0.63 (95\% CI: 0.38-1.04) for patients with FXI $<15 \%$ (vs. 0.55 ; $95 \%$ CI: $0.07-4.33$ in our study) and 0.49 (95\%: $0.30-0.80$ ) for patients with FXI 15 to <50\% (vs. $0.55 ; 95 \% \mathrm{CI}$ : $0.06-4.73$ in our study). ${ }^{17}$ Similarly, for VTE, our aHR was 0.45 (95\%: 0.06-3.47) compared with $0.26(0.08-0.84)$ for patients with $\mathrm{FXI}<50 \%$ in the previous study. ${ }^{17}$ While the point estimates are similar, we observed fewer events because of differences in population size (Clalit insures $~ 50 \%$ of the population, and MHS $\sim 25 \%$ and, consequently, the size of their FXI-deficient population was more than twice as large 
Table 2 10-year cumulative incidence, unadjusted and adjusted hazard ratios comparing incident severe bleeding and CRNSB among patients with FXI deficiency to their matched general population reference group

\begin{tabular}{|c|c|c|c|c|}
\hline & $N$ events & 10-year risk $(95 \% \mathrm{CI})$ & $\begin{array}{l}\text { Unadjusted HR } \\
(95 \% \mathrm{Cl})\end{array}$ & $\begin{array}{l}\text { Fully adjusted HR } \\
(95 \% \mathrm{Cl})\end{array}$ \\
\hline \multicolumn{5}{|l|}{ Severe bleeding } \\
\hline \multicolumn{5}{|l|}{ Severe FXI deficiency (<15\%) } \\
\hline Factor XI cohort, $n=209$ & 1 & $0.50 \%(0.00-1.50 \%)$ & $0.54(0.07-4.15)$ & $0.49(0.06-3.95)$ \\
\hline General population controls, $n=1,463$ & 13 & $1.60 \%(0.60-2.60 \%)$ & Ref. & Ref. \\
\hline \multicolumn{5}{|l|}{ Partial FXI deficiency ( 15 to $<50 \%$ ) } \\
\hline Factor XI cohort, $n=368$ & 7 & $2.50 \%(0.60-4.40 \%)$ & $5.38(2.00-14.43)$ & $5.27(1.91-14.52)$ \\
\hline General population controls, $n=2,576$ & 9 & $0.50 \%(0.20-0.90 \%)$ & Ref. & Ref. \\
\hline \multicolumn{5}{|l|}{ Overall FXI deficiency ( $<50 \%)$} \\
\hline Factor XI cohort, $n=577$ & 8 & $1.90 \%(0.50-3.20 \%)$ & $2.53(1.13-5.69)$ & $2.56(1.13-5.81)$ \\
\hline General population controls, $n=4,039$ & 22 & $0.90 \%(0.50-1.30 \%)$ & Ref. & Ref. \\
\hline \multicolumn{5}{|l|}{ Clinically relevant nonsevere bleeding } \\
\hline \multicolumn{5}{|l|}{ Severe FXI deficiency $(<15 \%)$} \\
\hline Factor $\mathrm{XI}$ cohort, $n=213$ & 21 & $13.80 \%(7.70-19.60 \%)$ & $1.55(0.97-2.49)$ & $1.53(0.94-2.47)$ \\
\hline General population controls, $n=1,491$ & 99 & $10.80 \%(8.50-13.10 \%)$ & Ref. & Ref. \\
\hline \multicolumn{5}{|l|}{ Partial FXI deficiency ( 15 to $<50 \%$ ) } \\
\hline Factor XI cohort, $n=373$ & 31 & $10.50 \%(6.60-14.30 \%)$ & $1.44(0.98-2.12)$ & $1.41(0.95-2.08)$ \\
\hline General population controls, $n=2,611$ & 151 & $8.40 \%(7.00-9.80 \%)$ & Ref. & Ref. \\
\hline \multicolumn{5}{|l|}{ Overall FXI deficiency $(<50 \%)$} \\
\hline Factor XI cohort, $n=586$ & 52 & $11.60 \%(8.30-14.80 \%)$ & $1.48(1.10-2.00)$ & $1.45(1.08-1.97)$ \\
\hline General population controls, $n=4,102$ & 250 & $9.20 \%(8.00-10.40 \%)$ & Ref. & Ref. \\
\hline
\end{tabular}

Abbreviations: $\mathrm{Cl}$, confidence interval; CRNSB, clinically relevant nonsevere bleeding; FXI, factor XI; HR, hazard ratio.

Note: Adjusted HR included: age (continuous), sex, district, socioeconomic status, smoking status, BMI (continuous), Deyo-Charlson Index (continuous), comorbidities, and drug exposures in the past 6 months and the HAS-BLED score.

Severe Bleeding,

Deficient (FXI<50\%) vs GPRG

Strata - GPRG - FXI deficient

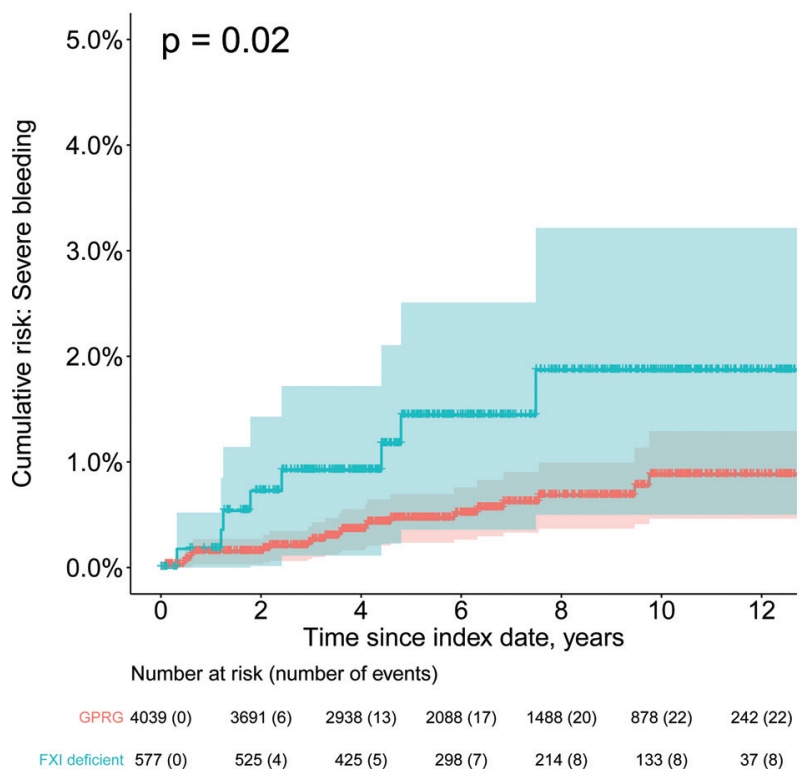

Clinically relevant non-severe bleeding, Deficient $(F X \mid<50 \%)$ vs GPRG

Strata - GPRG - FXI deficient

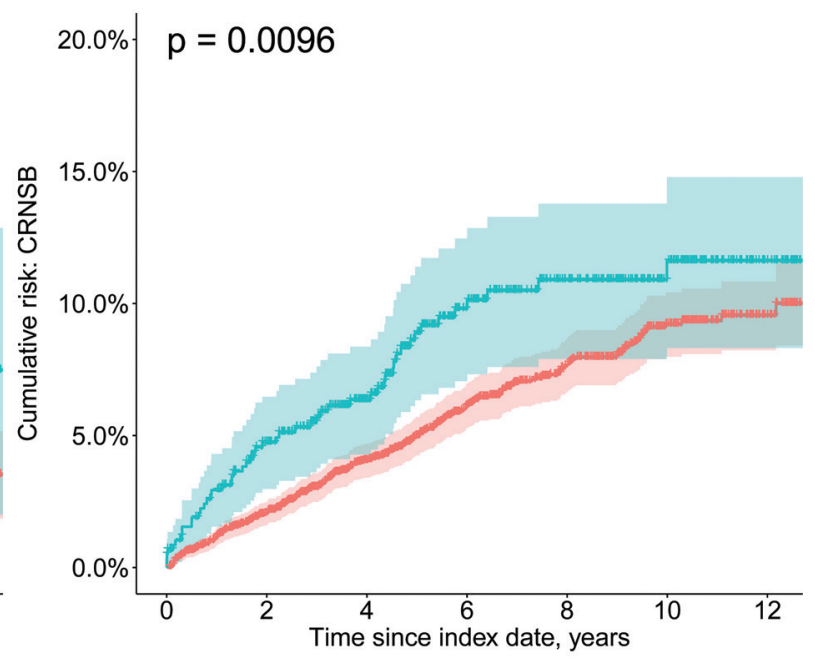

Number at risk (number of events)

GPRG $4102(0) \quad 3674(81) \quad 2868(150) \quad 1982(201) \quad 1400(229) \quad 804(247) \quad 219(249)$ FXl deficient $586(0) \quad 512(27) \quad 404(35) \quad 274(48) \quad 197(51) \quad 123(52) \quad 35(52)$

Fig. 1 Cumulative incidence of first severe bleeding and clinically relevant nonsevere bleeding among patients with factor XI deficiency ( $<50 \%)$ and their matched general population reference group. 
Table 3 10-year cumulative incidence, unadjusted and adjusted hazard ratios comparing incident CV and VTE events among patients with FXI deficiency to their matched general population reference group

\begin{tabular}{|c|c|c|c|c|}
\hline & $N$ events & 10-year risk $(95 \% \mathrm{CI})$ & $\begin{array}{l}\text { Unadjusted HR } \\
(95 \% \mathrm{Cl})\end{array}$ & $\begin{array}{l}\text { Fully adjusted HR } \\
(95 \% \mathrm{Cl})\end{array}$ \\
\hline \multicolumn{5}{|l|}{ CV events } \\
\hline \multicolumn{5}{|l|}{ Severe FXI deficiency $(<15 \%)$} \\
\hline Factor XI cohort, $n=208$ & 1 & $0.50 \%(0.00-1.60 \%)$ & $0.48(0.06-3.63)$ & $0.55(0.07-4.33)$ \\
\hline General population controls, $n=1,456$ & 15 & $1.80 \%(0.80-2.70 \%)$ & Ref. & Ref. \\
\hline \multicolumn{5}{|l|}{ Partial FXI deficiency ( 15 to $<50 \%$ ) } \\
\hline Factor XI cohort, $n=369$ & 1 & $0.30 \%(0.00-0.90 \%)$ & $0.86(0.11-6.90)$ & $0.55(0.06-4.73)$ \\
\hline General population controls, $n=2,583$ & 8 & $0.40 \%(0.10-0.70 \%)$ & Ref. & Ref. \\
\hline \multicolumn{5}{|l|}{ Overall FXI deficiency $(<50 \%)$} \\
\hline Factor $\mathrm{XI}$ cohort, $n=577$ & 2 & $0.40 \%(0.00-0.90 \%)$ & $0.61(0.14-2.58)$ & $0.55(0.13-2.36)$ \\
\hline General population controls, $n=4,039$ & 23 & $0.90 \%(0.50-1.20 \%)$ & Ref. & Ref. \\
\hline \multicolumn{5}{|l|}{ VTE } \\
\hline \multicolumn{5}{|l|}{ Severe FXI deficiency (<15\%) } \\
\hline Factor XI cohort, $n=212$ & 0 & $0 \%$ & $\mathrm{~N} / \mathrm{A}$ & $\mathrm{N} / \mathrm{A}$ \\
\hline General population controls, $n=1,484$ & 4 & $0.40 \%(0.00-0.90 \%)$ & $\mathrm{N} / \mathrm{A}$ & $\mathrm{N} / \mathrm{A}$ \\
\hline \multicolumn{5}{|l|}{ Partial FXI deficiency ( 15 to $<50 \%$ ) } \\
\hline Factor XI cohort, $n=371$ & 1 & $0.30 \%(0.00-0.90 \%)$ & $0.76(0.10-6.04)$ & $0.67(0.08-5.47)$ \\
\hline General population controls, $n=2,597$ & 9 & $0.50 \%(0.20-0.80 \%)$ & Ref. & Ref. \\
\hline \multicolumn{5}{|l|}{ Overall FXI deficiency (<50\%) } \\
\hline Factor XI cohort, $n=583$ & 1 & $0.20 \%(0.00-0.60 \%)$ & $0.53(0.07-4.09)$ & $0.45(0.06-3.47)$ \\
\hline General population controls, $n=4,081$ & 13 & $0.50 \%(0.20-0.70 \%)$ & Ref. & Ref. \\
\hline
\end{tabular}

Abbreviations: $\mathrm{Cl}$, confidence interval; CV, cardiovascular; FXI, factor XI; VTE, venous thromboembolism.

Note: Adjusted HR included: age (continuous), sex, district, socioeconomic status, smoking status, BMI (continuous), Deyo-Charlson Index (continuous), comorbidities, and drug exposures in the past 6 months and the $\mathrm{CHA}_{2} \mathrm{DS}_{2}$-VASc score.

\section{CV (Stroke/TIA/MI) events, Deficient (FXI<50\%) vs GPRG}

Strata + GPRG $=$ FXI deficient

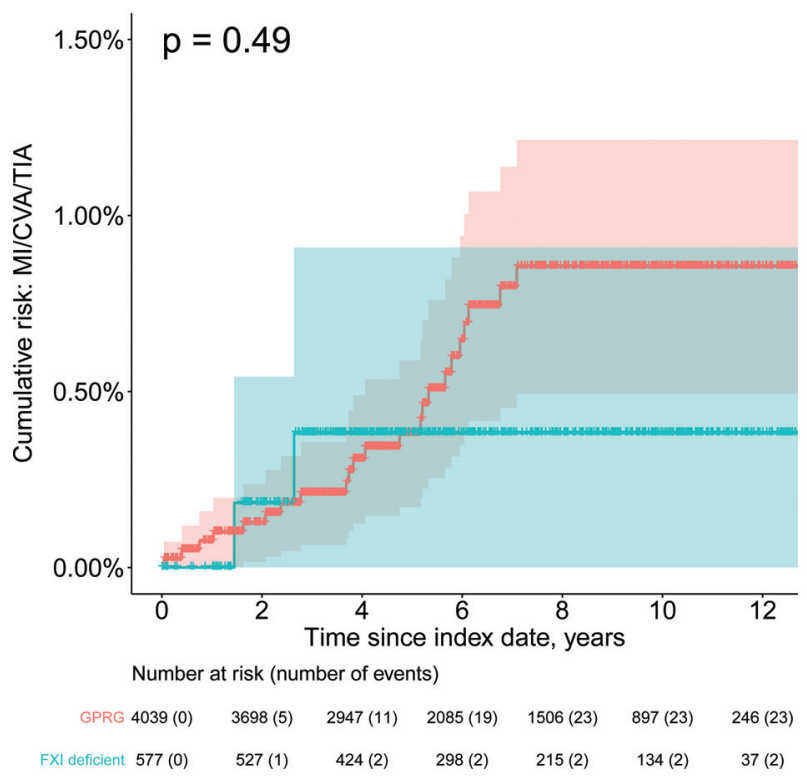

VTE events,

Deficient (FXI<50\%) vs GPRG

Strata $=$ GPRG + FXI deficient

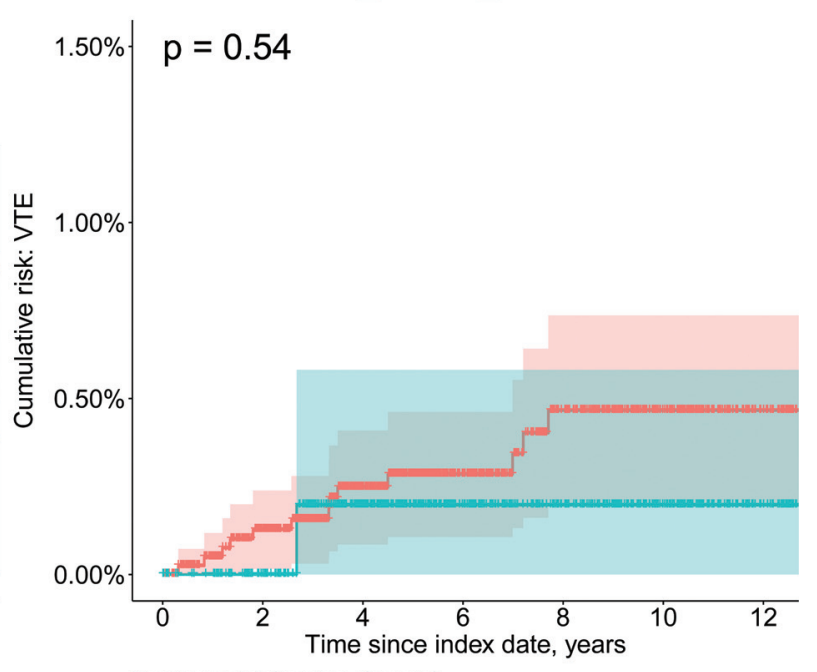

Number at risk (number of events)

GPRG $4081(0) \quad 3731(5) \quad 2968(9) \quad 2097(10) \quad 1507(13) \quad 896(13) \quad 247(13)$

FXI deficient $583(0) \quad 534(0) \quad 429(1) \quad 301(1) \quad 216(1) \quad 134(1) \quad 38(1)$

Fig. 2 Cumulative incidence of CV and VTE events among patients with factor XI deficiency $(<50 \%)$ and their matched general population reference group. CV, cardiovascular; VTE, venous thromboembolism. 
as ours) and study design (use of incident events in this study vs. prevalent events).

While the results of our study were in agreement with those of the study of Preis et $\mathrm{al}^{17}$ for CV and VTE events (the previous study did not examine bleeding risk), we found that the magnitude of the association between FXI and the outcomes was affected by choice of reference group. The magnitude of the association between FXI deficiencies was reduced for bleeding but increased for CV and VTE events when the reference group consisted of patients tested for FXI with normal FXI activity levels, compared with the GPRG, whose FXI levels were unknown but expected to be normal. Patients who undergo a FXI activity test, even if activity levels are found to be normal, are a heterogeneous cohort and are more likely to have other hematological disorders (e.g., VIII, IX, von Willebrand factor deficiencies) as compared with the general population, which may explain their elevated risk of bleeding. ${ }^{18,19,39}$ In our study, we found that patients with FXI normal test results were not only at increased risk of bleeding but were also at higher risk of $\mathrm{CV}$ and VTE events compared with the general population. This may be because many patients were likely tested for FXI deficiency prior to surgery, which itself is a risk factor for CV events and VTEs. In addition, patients with FXI $>50 \%$ include patients with elevated FXI levels, which also confers increased risk of thromboembolic events. Consequently, using patients who were tested for FXI as a reference group is likely to result in selection bias and an overestimation of the true association between FXI and CV/VTE outcomes.

The results of this study must be interpreted in the context of certain limitations. Given that patients underwent their first FXI activity test around the age of 40 to 45 years and we followed up most patients a median of 8 years, we observed few events. The mean age of stroke in Israel is 72.3 years ${ }^{40}$ and of MI is 66.6 years. ${ }^{41}$ While the point estimates for the association between FXI deficiency and CV and VTE events were consistent with those from other studies, we were limited in our ability to draw firm conclusions. Due to the small number of events observed, we were also not able to fully explore dose-response between the severity of FXI deficiency and outcomes. While the prevalence of FXI deficiency does not differ between males and females, ${ }^{10,42}$ most patients with FXI deficiency in this study were females between 35 and 45 years of age. Females may be more likely to undergo FXI testing because of abnormally heavy menstrual bleeding or workup care related to abortions, pregnancy, or childbirth. ${ }^{43}$ Due to the sample size, we did not explore and thus cannot rule out whether the relationship between FXI deficiency and outcomes differ by sex. The reason for FXI testing was also not available in our database. Future research should seek to better understand the motivation for FXI testing as being tested for FXI was associated with an increased risk of bleeding, CV, and VTEs. Lastly, we cannot rule out the strong possibility for residual confounding.

\section{Conclusion}

FXI deficiency is associated with an increased risk of severe bleeding and CRNSB. Our study also suggests that there may be a negative association between FXI deficiency and CV and VTE events. Additional studies are needed to further explore the relationship between FXI deficiency and bleeding as well as CV and VTE events. These results could have implications for the development of FXI target therapy to inhibit CV events without increased bleeding risk.

\section{What is known about this topic?}

- FXI deficiency is characterized by prolonged bleeding after trauma or surgery and patients rarely exhibit spontaneous bleeding typical of other hemophilia diseases.

- Some studies have reported that low levels of FXI do not predict clinical bleeding severity and there is also some evidence that FXI deficiency may be protective against some thromboembolic events.

\section{What does this paper add?}

- Patients with FXI deficiency are at increased risk of bleeding compared with the general population.

- Further research is warranted to explore the lower risk of CV and VTE among patients with FXI deficiency compared with the general population.

\section{Author Contributions}

Conceptualization, methodology, or analysis: S.S.M., G.C., Y.G.N., D.C., M-.D.W., A.R.S., L.M., O.S., J.J.J.; writingoriginal draft preparation: S.S.M., G.C., J.J.J.; final approval: S.S.M., G.C., Y.G.N., D.C., M-.D.W., A.R.S., L.M., O.S., J.J.J.; accountability: S.S.M., G.C., Y.G.N., D.C., M-.D.W., A.R.S., L.M., O.S., J.J.J.

\section{Funding}

This work was supported by Regeneron Pharmaceuticals, Tarrytown, New York, United States. The funding source was involved in study design and in the writing of the report.

Conflict of Interest

Y.G.N., D.C., M-.D.W., A.R.S., L.M., and J.J.J. are employees of Regeneron. S.S.M., G.C., O.S. have no declarations of interest.

\section{References}

1 Rosenthal RL, Dreskin OH, Rosenthal N. New hemophilia-like disease caused by deficiency of a third plasma thromboplastin factor. Proc Soc Exp Biol Med 1953;82(01):171-174

2 Gomez K, Bolton-Maggs P. Factor XI deficiency. Haemophilia 2008;14(06):1183-1189

3 Rapaport SI, Proctor RR, Patch MJ, Yettra M. The mode of inheritance of PTA deficiency: evidence for the existence of major PTA deficiency and minor PTA deficiency. Blood 1961;18(02): 149-165

4 Asakai R, Chung DW, Davie EW, Seligsohn U. Factor XI deficiency in Ashkenazi jews in Israel. N Engl J Med 1991;325(03):153-158

5 Duga S, Salomon O. Factor XI deficiency. Semin Thromb Hemost 2009;35(04):416-425 
6 Shpilberg O, Peretz H, Zivelin A, et al. One of the two common mutations causing factor XI deficiency in Ashkenazi Jews (type II) is also prevalent in Iraqi Jews, who represent the ancient gene pool of Jews. Blood 1995;85(02):429-432

7 Seligsohn U. High gene frequency of factor XI (PTA) deficiency in Ashkenazi Jews. Blood 1978;51(06):1223-1228

8 Salomon O, Steinberg DM, Seligshon U. Variable bleeding manifestations characterize different types of surgery in patients with severe factor XI deficiency enabling parsimonious use of replacement therapy. Haemophilia 2006;12(05):490-493

9 Seligsohn U. Factor XI deficiency in humans. J Thromb Haemost 2009;7(Suppl 1):84-87

10 Bolton-Maggs PH. Factor XI deficiency-resolving the enigma? Hematology Am Soc Hematol Educ Program 2009;2009(01):97-105

11 Rimon A, Schiffman S, Feinstein DI, Rapaport SI. Factor XI activity and factor XI antigen in homozygous and heterozygous factor XI deficiency. Blood 1976;48(02):165-174

12 Peyvandi F, Palla R, Menegatti M, et al; European Network of Rare Bleeding Disorders Group. Coagulation factor activity and clinical bleeding severity in rare bleeding disorders: results from the European Network of Rare Bleeding Disorders. J Thromb Haemost 2012;10(04):615-621

13 Santoro C, Di Mauro R, Baldacci E, et al. Bleeding phenotype and correlation with factor XI (FXI) activity in congenital FXI deficiency: results of a retrospective study from a single centre. Haemophilia 2015;21(04):496-501

14 Salomon O, Steinberg DM, Koren-Morag N, Tanne D, Seligsohn U. Reduced incidence of ischemic stroke in patients with severe factor XI deficiency. Blood 2008;111(08):4113-4117

15 Salomon O, Steinberg DM, Zucker M, Varon D, Zivelin A, Seligsohn U. Patients with severe factor XI deficiency have a reduced incidence of deep-vein thrombosis. Thromb Haemost 2011;105 (02):269-273

16 Girolami A, Peroni E, Girolami B, Ferrari S, Lombardi AM. Congenital factor XI and factor VII deficiencies assure an apparent opposite protection against arterial or venous thrombosis: an intriguing observation. Hematology 2016;21(08):486-489

17 Preis M, Hirsch J, Kotler A, et al. Factor XI deficiency is associated with lower risk for cardiovascular and venous thromboembolism events. Blood 2017;129(09):1210-1215

18 Yang DT, Flanders MM, Kim H, Rodgers GM. Elevated factor XI activity levels are associated with an increased odds ratio for cerebrovascular events. Am J Clin Pathol 2006;126(03):411-415

19 Meijers JC, Tekelenburg WL, Bouma BN, Bertina RM, Rosendaal FR. High levels of coagulation factor XI as a risk factor for venous thrombosis. N Engl J Med 2000;342(10):696-701

20 Shalev V, Chodick G, Goren I, Silber H, Kokia E, Heymann AD. The use of an automated patient registry to manage and monitor cardiovascular conditions and related outcomes in a large health organization. Int J Cardiol 2011;152(03):345-349

21 Goldshtein I, Chandler J, Shalev V, et al. Osteoporosis in the community: findings from a novel computerized registry in a large health organization in Israel. J Aging Res Clin Pract 2015;4 (01):59-65

22 Chodick G, Heymann AD, Shalev V, Kookia E. The epidemiology of diabetes in a large Israeli HMO. Eur J Epidemiol 2003;18(12):1143-1146

23 Weitzman D, Chodick G, Shalev V, Grossman C, Grossman E. Prevalence and factors associated with resistant hypertension in a large health maintenance organization in Israel. Hypertension 2014;64(03):501-507

24 Israel Center for Disease Control MoH. Israel National Cancer Registry. Accessed August 27, 2021 at: https://www.health.gov. il/English/MinistryUnits/HealthDivision/Icdc/Icr/Pages/default. aspx

25 Bolton-Maggs PH, Young Wan-Yin B, McCraw AH, Slack J, Kernoff $\mathrm{PB}$. Inheritance and bleeding in factor XI deficiency. Br J Haematol 1988;69(04):521-528
26 McCormick N, Bhole V, Lacaille D, Avina-Zubieta JA. Validity of diagnostic codes for acute stroke in administrative databases: a systematic review. PLoS One 2015;10(08):e0135834

27 Cunningham A, Stein CM, Chung CP, Daugherty JR, Smalley WE, Ray WA. An automated database case definition for serious bleeding related to oral anticoagulant use. Pharmacoepidemiol Drug Saf 2011;20(06):560-566

28 Wahl PM, Rodgers K, Schneeweiss S, et al. Validation of claims-based diagnostic and procedure codes for cardiovascular and gastrointestinal serious adverse events in a commercially-insured population. Pharmacoepidemiol Drug Saf 2010;19(06):596-603

29 Sanfilippo KM, Wang T-F, Gage BF, Liu W, Carson KR. Improving accuracy of International Classification of Diseases codes for venous thromboembolism in administrative data. Thromb Res 2015;135(04):616-620

30 Israel Central Bureau of Statistics. Characterization and classification of geographic units by the socio-economic level of the population 2013. Publication No. 1530. 2013. Accessed August 21, 2021 at: https://www.cbs.gov.il/en/publications/Pages/2017/ Characterization-and-Classification-of-Geographical-Units-by-theSocio-Economic-Level-of-the-Population-2013.aspx

31 Deyo RA, Cherkin DC, Ciol MA. Adapting a clinical comorbidity index for use with ICD-9-CM administrative databases. J Clin Epidemiol 1992;45(06):613-619

32 Pisters R, Lane DA, Nieuwlaat R, de Vos CB, Crijns HJ, Lip GY. A novel user-friendly score (HAS-BLED) to assess 1-year risk of major bleeding in patients with atrial fibrillation: the Euro Heart Survey. Chest 2010;138(05):1093-1100

33 Lip GY, Nieuwlaat R, Pisters R, Lane DA, Crijns HJ. Refining clinical risk stratification for predicting stroke and thromboembolism in atrial fibrillation using a novel risk factor-based approach: the euro heart survey on atrial fibrillation. Chest 2010;137(02):263-272

34 Kumamaru H, Judd SE, Curtis JR, et al. Validity of claims-based stroke algorithms in contemporary Medicare data: reasons for geographic and racial differences in stroke (REGARDS) study linked with medicare claims. Circ Cardiovasc Qual Outcomes 2014;7(04):611-619

35 Cutrona SL, Toh S, Iyer A, et al. Validation of acute myocardial infarction in the Food and Drug Administration's Mini-Sentinel program. Pharmacoepidemiol Drug Saf 2013;22(01):40-54

36 Peyvandi F, Menegatti M. Treatment of rare factor deficiencies in 2016. Hematology (Am Soc Hematol Educ Program) 2016;2016 (01):663-669

37 Bolton-Maggs PH, Patterson DA, Wensley RT, Tuddenham EG. Definition of the bleeding tendency in factor XI-deficient kindreds-a clinical and laboratory study. Thromb Haemost 1995;73 (02):194-202

38 Franchini M, Manzato F, Salvagno GL, Montagnana M, Lippi G. The use of desmopressin in congenital factor XI deficiency: a systematic review. Ann Hematol 2009;88(10):931-935

39 Doggen CJ, Rosendaal FR, Meijers JC. Levels of intrinsic coagulation factors and the risk of myocardial infarction among men: opposite and synergistic effects of factors XI and XII. Blood 2006; 108(13):4045-4051

40 Bornstein NM, Aronovich BD, Karepov VG, et al. The Tel Aviv Stroke Registry. 3600 consecutive patients. Stroke 1996;27(10):1770-1773

41 Plakht Y, Shiyovich A, Gilutz H. Predictors of long-term (10-year) mortality postmyocardial infarction: age-related differences. Soroka Acute Myocardial Infarction (SAMI) Project. J Cardiol 2015;65(03):216-223

42 UpToDate. Factor XI (eleven) deficiency. Accessed December 14, 2020 at: https://www.uptodate.com/contents/factor-xi-elevendeficiency?search $=$ Factor\%20XI\%20deficiency\%20sex\&source $=$ search_result\&selectedTitle $=1 \sim 150 \&$ usage_type $=$ default $\&$ display_rank $=1$

43 Kadir RA, Economides DL, Lee CA. Factor XI deficiency in women. Am J Hematol 1999;60(01):48-54 